\title{
Recent Modalities in Management of Early Complications of Sleeve Gastrectomy
}

\author{
E.S.Radwan, H.K.El Sweny, E.M.Oraby and I.M.Amer
}

General surgery, Dept.,Faculty of Medicine, Benha Univ., Benha, Egypt

Email:ddoocc.ia@gmail.com

\begin{abstract}
Background: One of the most common operation for weight loss is the laparoscopic sleeve gastrectomy which are an effective and relatively safe. Laparoscopic sleeve gastrectomy is superior in comparison to other restrictive procedures due to the decrease of large parts of ghrelin producing stomach mass. Aim of the Work: to outline recent modalities in management of early complications of sleeve gastrectomy. This study was performed at Benha university hospitals and Kasr El Aini University Hospitals on 30 early complicated cases post sleeve gastrectomy at the period from 1/1/2018 to 30/6/2018. Patients and Methods: This study was performed at Benha university hospitals at the period from 1/1/2018 to $30 / 6 / 2018$ on 30 early complicated cases post sleeve gastrectomy. Consent of the patients was taken and data were collected included: age, sex, BMI, and comorbid conditions at the time of admission. Results: According to the co morbidities, eight of the studied cases (26.7\%) suffered from hypertension, and 22 others $(73.3 \%)$ didn't suffer from hypertension, also half of the studied cases $(50.0 \%)$ suffered from diabetes. As regard the complications that the patients suffered after the operation in our study, 7 patients (23.3\%) suffered of Tachycardia, 5 patients (16.7\%) suffered of Vomiting, 4 patients (13.3\%) suffered of Hemorrhage, Leakage and Hematemsis, 3 patients (10.0\%) suffered of Wound infection, 2 patients (6.7\%) suffered of Gastric stenosis, Conversion to open and Melena. On the other side, none of the patients suffered from DVT, Gastric volvulus, pulmonary embolism and Mortality. Conclusion: LSG is one of the most effective surgical procedures in weight loss and is considered safe if its complications are diagnosed and managed early and we recommended to do this study on largenumber of patients to support our results and to know more about the sleeve gastrectomy complications to manage them as early as possible to get benefit from sleeve gastrectomy and to be used on a wide range.
\end{abstract}

Key words: Early Complications of Sleeve Gastrectomy, Management.

\section{Introduction}

Obesity is a worldwide epidemic associated with diseases such as diabetes mellitus and cardiovascular disease. Current methods for weight loss are not very effective, particularly for those with morbid obesity. Surgical therapy may be recommended for those with a BMI $\geq 40 \mathrm{~kg} / \mathrm{m} 2$, or BMI $\geq 35 \mathrm{~kg} / \mathrm{m} 2$ with co-morbidities. This therapy can produce significant weight loss and improve/resolve co-morbidities including hypertension and hyperlipidemia [1].

The introduction of the laparoscopy has widely proved its advantages over the open approach in various types of surgery. The same advantages are found in bariatric surgery with the difference that the morbid obese patients have more risks than non-obese patients for cardiopulmonary complications, infections, and woundrelated complications [2].

Pneumoperitoneum has negative effects on the respiratory and cardiovascular systems. Morbid obese patients have a baseline intrabdominal pressure approximately equal to 2-3 times that of a non-obese. The intraperitoneal introduction of $\mathrm{CO} 2$ leads to a systemic absorption of $\mathrm{CO}$, a reduction of cardiac preload due to venous compression reducing intraoperative urine output, a decrease of lung compliance, increase of airway pressure, and cardiac function impairment. The optimization of anesthesiologic procedures may minimize these adverse changes [3].

The laparoscopic sleeve gastrectomy consists of a partial gastrectomy with removal of the greater curvature and fundus. This creates a small sleeve or tube of residual stomach, which includes the lesser curvature and antrum. It is typically performed laparoscopically with 5 or 6 trocars. The anesthesiologist inserts a $32-48$ French bougie (thin cylinder) via the esophagus, into the stomach along the lesser curvature. The stomach is transected using staplers beginning approximately $6 \mathrm{~cm}$ proximal to the pylorus. Staplers are fired cephalad in close proximity to the bougie and parallel to the lesser curvature until the angle of His is reached. The "comma-shaped" specimen is then extracted [4].

Sleeve Gastrectomy seems to have a smaller risk of complications, but the potential complications can be as severe as those associated with other techniques. Due to the long staple line and the altered intragastric pressure, sleeve gastrectomy is specifically associated with two significant complications: hemorrhage and leakage. The most common complication after sleeve gastrectomy is a gastric leak, which most commonly occurs at the upper staple line near the gastroesophageal junction. This complication, if not identified and treated quickly and aggressively, may lead to severe abdominal sepsis, which might progress to either a chronic gastric fistula or to multiorgan failure and patient demise [5].

Early technical, postoperative complications after sleeve gastrectomy include postoperative staple line leaks or iatrogenic perforation, obstruction and/or bleeding. Although the rate of these shared complications is low and many can be managed nonoperatively, it is imperative for the bariatric surgeon to have a robust understanding of the presentation of these potentially life-threatening complications to enable rapid detection, diagnosis, and operative management when indicated to minimize morbidity and mortality [6].

Interventional options include surgery (laparoscopy or laparotomy with abdominal washout, abdominal drainage close to the staple line, and feeding jejunostomy), endoscopic 
procedures (self-expandable metallic stents (SEMS), clips, biological glue, pigtail drains, and T-tube gastrostomy drain), and radiological procedures (percutaneous drainage). The management of the leak depends on the patient's clinical condition [7].

\section{Aim of the Work}

Aim of the work is to outline recent modalities in management of early complications of sleeve gastrectomy.

\section{Patients and Methods}

This study was performed at Benha university hospitals at the period from $1 / 1 / 2018$ to $30 / 6 / 2018$ on 30 early complicated cases post sleeve gastrectomy.

Consent of the patients was taken and data were collected included: age, sex, BMI, and comorbid conditions at the time of admission.

This study was conducted on early complicated cases post sleeve gastrectomy.

We excluded uncomplicated cases with late complications post sleeve gastrectomy.

The patients were subjected to:

Laboratory investigations: Complete blood picture, blood glucose level, liver and kidney function. Pulmonary function tests, chest radiography, abdomen, and pelvis ultrasound also were done.

Intraoperative and early postoperative complications such as bleeding, leakage, splenic injury, vomiting, wound infection, malrotation, and mortality were recorded.

\section{Methodology:}

Patients were encouraged to start oral sips on the first post-operative day, progressing gradually to soft food. Tachycardia $\left(>100\right.$ beats/min), fever $\left(\geq 38.5^{\circ} \mathrm{C}\right)$, abdominal pain, tachypnea $(>20$ breaths $/ \mathrm{min})$, or decreased urinary output despite good hydration were handled with a high index of suspicion. Laboratory findings of C-reactive protein (CRP)
$>150 \mathrm{mg} / \mathrm{l}$ and leukocytosis $>11,000$ cells/ $\mu$ l were suggestive of complications. CRP level and complete blood count were routinely checked on the first and fifth post-operative day.

Bleeding was suspected by a post-operative hemoglobin level drop of at least $2 \mathrm{~g} / \mathrm{dl}$, aided by CT evidence.

Leakage was defined as trickling of gastric and/or intestinal contents outside the visceral lumen. It was diagnosed radiologically by detection of contrast outside the lumen in contrast-enhanced computed tomography (CT) scans or gastrografin swallows. This may have been aided by detection of abscess cavity, air foci in the vicinity of operative field, or stranded mesentery on CT scans.

Procedures done for these patients to manage the complications as: Mega stent in cases of leakage and gastric stenosis. Laparoscopic clips applying in cases of bleeding and exploration in cases of peritonitis Bleeding was controlled using surgical intervention in hemodynamically unstable patients, where hematoma evacuation, over sewing, and drainage were done. Otherwise, we resorted to conservative methods (fluid resuscitation \pm blood transfusion). Leakage was an indication for reoperation.

\section{Statistical Analysis}

Data were collected, revised, coded and entered to the Statistical Package for Social Science (IBM SPSS) version 20. The qualitative data were presented as number and percentages while quantitative data were presented as mean, standard deviations and ranges when their distribution found parametric.

The confidence interval was set to $95 \%$ and the margin of error accepted was set to $5 \%$. So, the p-value was considered significant as $\mathrm{P}<0.05=$ significant $(\mathrm{S})$.

\section{Results}

Table (1) Distribution of the studied cases according to Age, Sex and BMI.

\begin{tabular}{llc}
\hline & & No.=30 \\
\hline \multirow{2}{*}{ Sex } & Male & $14(46.7 \%)$ \\
& Female & $16(53.3 \%)$ \\
Age & Mean \pm SD & $38.87 \pm 9.73$ \\
& Range & $25-55$ \\
BMI & Mean \pm SD & $46.80 \pm 7.98$ \\
& Range & $34-60$ \\
\hline
\end{tabular}

Table (2): Distribution of the studied cases according to Hypertension and Diabetes

\begin{tabular}{llcc}
\hline Comorbidities & & No. & \% \\
\hline \multirow{2}{*}{ Hypertension } & -ve & 22 & $73.3 \%$ \\
\multirow{2}{*}{ Diabetes } & +ve & 8 & $26.7 \%$ \\
& -ve & 15 & $50.0 \%$ \\
\hline
\end{tabular}

Table (3) Distribution of the studied cases according to Complications after surgery

\begin{tabular}{ccc}
\hline Complications after surgery & No. & \% \\
\hline Tachycardia & 7 & $23.3 \%$ \\
Vomiting & 5 & $16.7 \%$ \\
Hemorrhage & 4 & $13.3 \%$ \\
Leakage & 4 & $13.3 \%$ \\
Hematemsis & 4 & $13.3 \%$ \\
\hline
\end{tabular}




$\begin{array}{ccc}\text { Wound infection } & 3 & 10.0 \% \\ \text { Melena } & 2 & 6.7 \% \\ \text { Conversion to open } & 2 & 6.7 \% \\ \text { Gastric stenosis } & 2 & 6.7 \% \\ \text { DVT } & 0 & 0.0 \% \\ \text { Gastric volvulus } & 0 & 0.0 \% \\ \text { Pulmonary embolism } & 0 & 0.0 \% \\ \text { Mortality } & 0 & 0.0 \%\end{array}$

Four patients (two men and two women) with median age of 44 years and a median BMI of $38.3 \mathrm{~kg} / \mathrm{m}^{2}$ were presented by postoperative Intraperitoneal bleeding, one of them presented a hypovolemic shock in the immediate postoperative period, drain amount was about $1100 \mathrm{cc}$ blood and hematocrit drop to $20 \%$, patient was hemodynamically unstable with rising heart rate despite blood transfusion and fluid resuscitation, urgent surgical revision was carried out through laparoscopic approach with control of bleeding source by oversewing running sutures.

The other three patients were hypertensive with uncontrolled HTN and presented by bleeding, conservative treatment was done, patients improved and bleeding stopped on fluid resuscitation, blood transfusion, control of blood pressure and daily montoring of $\mathrm{CBC}$ and hemodynamic state.

Two patients (a man and a woman) with a median age of 48 years and a median BMI of $42,73 \mathrm{~kg} / \mathrm{m}^{2}$ were presented with melena started from day two postoperative, both of them were hypertensive with uncontrolled HTN, both of them underwent upper GI endoscopy for control of the intraluminal source of bleeding and melena stopped, in the two patients melena stopped on conservative treatment, antihemorrhagic drugs and control of blood pressure.

Four patients (three women and a man) with a median age of 32 years and a median BMI of $36.1 \mathrm{~kg} / \mathrm{m}^{2}$ were presented with postoperative leakage. The four patients were diabetic with uncontrolled DM, conservative treatment was started for them (NPO, IV broad-spectrum antibiotics, IV fluids and control of blood sugar), Percutaneous drainage under ultrasound or computed tomography guidance with pigtail catheter placement was performed for abdominal collections. Then an upper gastrointestinal series with water-soluble contrast media and esophagogastro-duodenoscopy were performed for diagnosis of the diagnosis of leak after infection under control, and the site and size of the leak were recorded during esophagoduodenoscopy. Fully coverd Taewoong Niti-S esophageal stents were used to cover the site of leak and allow oral diet for patient. All patients improved with complete leak closure within 6-8 weeks

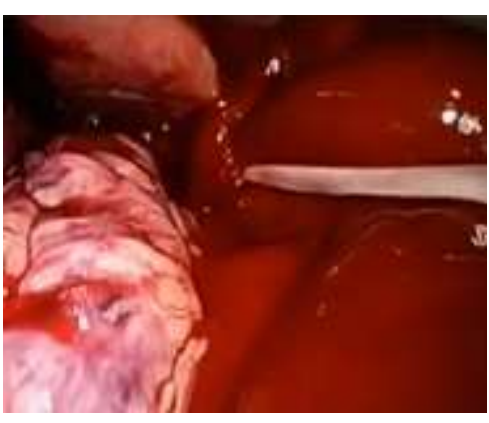

Fig. (1) Mesocolic hematoma

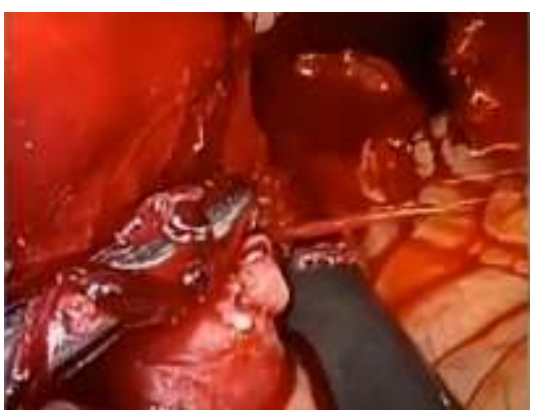

Fig. (2) Identification of bleeding source

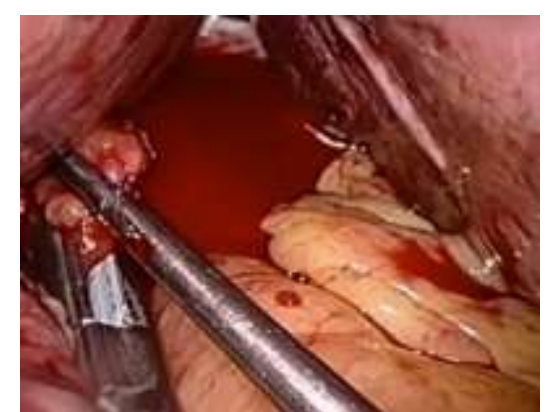

Fig. (3) Control of bleeding source

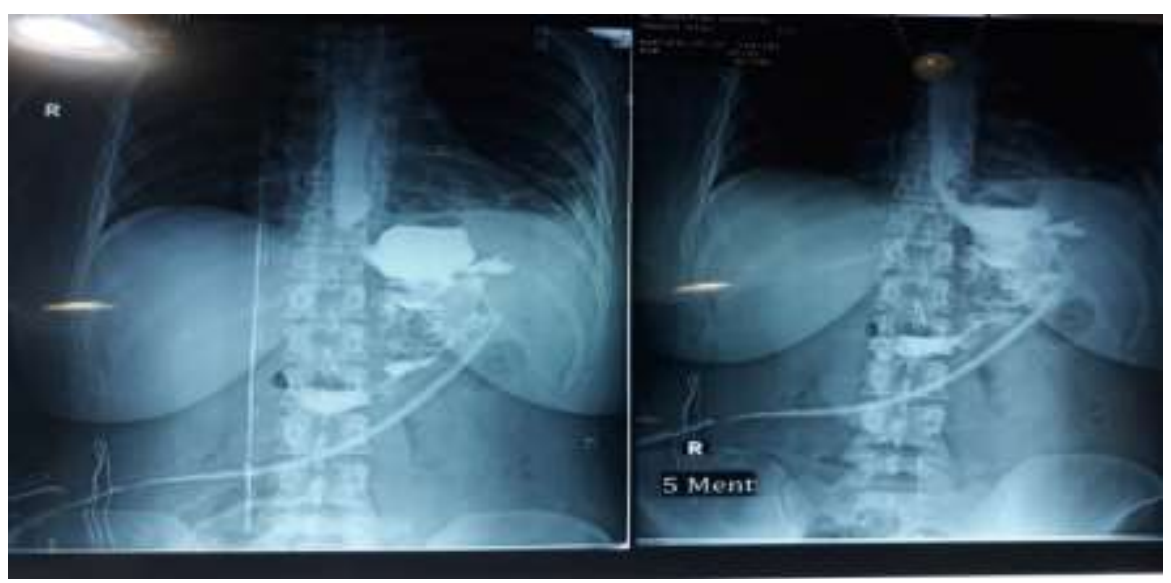

Fig. (4) Intra-abdominal leakage by gastrografin study. 


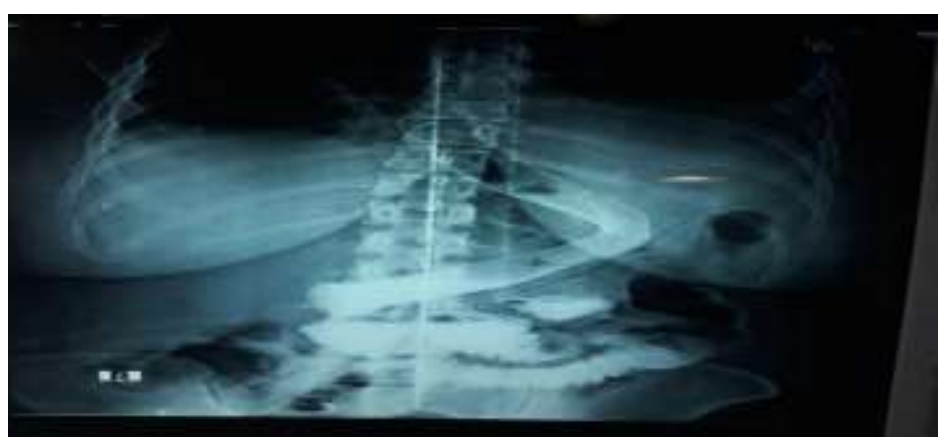

Fig. (5) Leakage stopped after endoscopic insertion of gastric stent.

Four patients (three women and a man) with a median age of 48 years and a median BMI of $46.8 \mathrm{~kg} / \mathrm{m}^{2}$ were presented with vomiting at the first two postoperative days, the rate of vomiting was from three to five times daily, patients were bedridden with silent intestinal sounds by auscultation, it's most probably due to postoperative ileus, they improved on antiemetic drugs, movement and replacement of electrolyte balance.

Three patients (two women and a man) with a median age of 49 years and a median BMI of $41.33 \mathrm{~kg} / \mathrm{m}^{2}$ were presented with postoperative wound infection, they were diabetic with uncontrolled DM, they improved on repeated dressings, proper IV antibiotics, and control of blood sugar.

\section{Discussion}

Laparoscopic sleeve gastrectomy (LSG) has gained popularity because it is considered a technically less demanding operation and has potential benefits as the intestinal passage is left intact, allowing the endoscopy of the remaining stomach and the access to the duodenum, and there are no bowel diversions, thus eliminating the risk of internal hernia [8]. Recent studies reported a substantial decrease in LSG complication rates. However, complications after LSG can be severe and even fatal in some cases [9].

This study was performed at Benha university hospitals and Kasr El Aini University Hospitals on 30 early complicated cases post sleeve gastrectomy at the period from $1 / 1 / 2018$ to $30 / 6 / 2018$.

Our study included 14 males represented $46.7 \%$ and 16 females represented $53.3 \%$, with an average age of 38.87 and a standard deviation of 9.73 with the lowest age of patients was 25 years and the oldest was 55 years, and the average BMI was 46.80 with a Standard deviation 7.98

In the study, eight of the studied cases (26.7\%) suffered from hypertension, and 22 others $(73.3 \%)$ didn't suffer from hypertension. Also half of the studied cases $(50.0 \%)$ suffered from diabetes.

Blood pressure measurements showed that for these patients, systolic and diastolic pressure was reduced by about 8.3 and $5.9 \mathrm{mmHg}$, respectively, at 10 days after the surgery and the reduction was maintained over the followup period.

Most of diabetic patients needed lower antidiabetic drug dose, in agreement with our results Bayham et al. [10] in a study reported that approximately $83 \%$ of sleeve gastrectomy patients remained off their diabetes medication.
Complications of LSG may be fatal if not diagnosed early and managed well. Various complications are reported following LSG, including leaks, bleeding, strictures, and nutritional deficiencies. The reported postoperative complication rates vary between 1 and $29 \%$ [11].

As regard the complications that the patients suffered after the operation in the study, 7 patients $(23.3 \%)$ suffered of Tachycardia, 5 patients $(16.7 \%)$ suffered of Vomiting, 4 patients (13.3\%) suffered of Hemorrhage, Leakage and Hematemsis, 3 patients $(10.0 \%)$ suffered of Wound infection, 2 patients $(6.7 \%)$ suffered of Gastric stenosis, Conversion to open and Melena. On the other side, none of the patients suffered from DVT, Gastric volvulus, pulmonary embolism and Mortality.

Mittermair et al. [5] reported that the postoperative bleeding rate in their study was 3.3\% and Sakran et al. [11] reported it to be $2.1 \%$. De Angelis et al. [12] reported a bleeding rate of $1.9 \%$ from splenic injury and suture line. In the current study bleeding were due to of staple line bleeding. However, there are other sources of bleeding, such as the omentum, the short gastric vessels, and the abdominal wall

Although in LSG no anastomosis is created, the long divided staple line carries a marked risk of leak that varies between 0 and $7 \%$ in primary procedures, and up to $20 \%$ in revisional surgery. The vast majority of leaks in LSG $(85.7 \%)$ occur in the proximal sleeve over the last staple firing, near the angle of His [13]. Some factors may share in leakage at that site as (a) insufficient staple height, as the gastric wall thickness varies from the antral to the cardial region; (b) tearing at the top of the staple line; (c) staple gun failure, increasing the risk of leakage at any point in the gastric division; and (d) ischemia from gastric tube weakness due to the stapling of branches arising from the left gastric artery [14].

According to a German multicentric observational study male sex and BMI between 50 and $59.9 \mathrm{~kg} / \mathrm{m} 2$ increase the risk for leakage by 2.5 and $1.6 \%$, respectively [15]. This comes in agreement with our results where patients with preoperative BMI more than 40 had higher risk of complications.

Among the eight hypertensive patients 3 patients had early post-operative bleeding. Hypertension is associated with increased peripheral vascular resistance and atherosclerosis, which lead to vascular remodeling [16]. We suspect that changes in the vascular histology and increased stiffness of the small vessels may have caused 
disturbances during stapler firing or ligating by the energy device.

Our study showed $13.3 \%$ with leakage while Boeker et al. [17]; their study showed a step line leakage of $2.1 \%$, Sakran et al. [18] showed $2.5 \%$, but it was best than ours in the study of Rondelli, et al. [8] which was $0.7 \%$ and the study of Noe [19] which was $1 \%$. This difference between studies may be related to the included criteria and to the different sample size.

Modification of surgical technique aims at reducing post-operative complications. Staple-line reinforcement is an example, with controversy of ideas regarding its efficacy. Some authors attributed a shorter operative time and fewer complications, including leakage, after LRYGB to the use of buttressing materials [20].

Others linked a less bleeding rate along the staple line to the use of Bioabsorbable sutures in LSG [21]. Other studies showed however no clear benefit with the use of buttressing materials [22].

\section{Conclusion}

Laparoscopic sleeve gastrectomy is one of the most effective surgical procedures in weight loss and is considered safe if its complications are diagnosed and managed early. Close clinical observation is crucial to detect and evaluate complications early in order to propose the best medical or surgical treatment in these patients.

\section{References}

[1] L.Kurukulasuriya, S.Stas, G. Lastra, C.Manrique J.Sowers. (2011): "Hyper-tension in obesity", Med Clin North Am; Vol. 95 (5), PP. (903-917), 2011.

[2] J.Reoch, S.Mottillo, A.Shimony, K.Filion N.Christou. "Safety of laparoscopic vs open bariatric surgery: a systematic review and metaanalysis", Arch Surg; Vol. 146 (11), PP. (1314-1322), 2011.

[3] T.Gaszynski, T.Szewczyk. "The influence of laparoscopic vs. open gastric bypass on hemodynamic function in morbidly obese patients during general anesthesia", Wideochir Inne Tech Malo Inwazyjne; Vol. 9 (1), PP. (83-88), 2014.

[4] D.P.Katz, S.R.Lee, A.C.Nachiappan, M.Willis C.Bray. "Laparoscopic sleeve gastrectomy: a guide to postoperative anatomy and complications", Abdom Imaging; Vol. 36, PP. (363-371), 2011.

[5] R.Mittermair, R.Sucher, A.Perathoner "Results and complications after laparoscopic sleeve gastrectomy", Surg Today; Vol. 44, PP. (1307-1312), 2014.

[6] NT.Nguyen, B.Nguyen, A.Gebhart S.Hohmann. Changes in the makeup of bariatric surgery: a national increase in use of laparoscopic sleeve gastrectomy, J Am Coll Surg; 216, PP. (252-257), 2013.

[7] X.De Aretxabala, J.Leon, G.Wiedmaier, I.Turu and C.Ovalle. "Gastric leak after sleeve gastrectomy: analysis of its management", Obes Surg; Vol. 21, PP. (1232-1237), 2011.

[8] F.Rondelli, W.Bugiantella, M.C.Vedovati, E.Mariani, R.C.Balzarotti, S.Federici. Laparoscopic gastric bypass versus laparoscopic sleeve gastrectomy: a retrospective multicenter comparison between early and long-term post-operative outcomes. Int J Surg; Vol. 37, PP. (36-41), 2017.

[9] M.Bashah, N.Khidir, M.EL-Matbouly. Management of leak after sleeve gastrectomy: outcomes of 73 cases, treatment algorithm and predictors of resolution. Obes Surg; Vol. 30, PP. (515-520, 2020.

[10] B.Bayham, F.Greenway, D.Bellanger, C.O'Neil. Early resolution of type 2 diabetes seen after Rouxen-Y gastric bypass and vertical sleeve gastrectomy. Diabetes Technol Ther.; Vol. 14(1), PP. (30-4), 2012.

[11] N.Sakran, A.Razielm, O.Goitein, A.Szold, D.Goitein. Laparoscopic sleeve gastrectomy for morbid obesity in 3003 patients: results at a highvolume bariatric center. Obes Surg; Vol. 26: PP. (2045-2050), 2016.

[12] F.De Angelis, M.Abdelgawad, M. Rizzello, C.Mattia, G.Silecchia. Perioperative hemorrhagic complications after laparoscopic sleeve gastrectomy: four-year experience of a bariatric center of excellence. Surg Endosc; Vol. 31: PP. (3547-3551), 2017.

[13] A.Burgos, I.Braghetto, A.Csendes A. Gastric leak after laparoscopic-sleeve gastrectomy for obesity. Obes Surg; Vol. 19, (1672-1677), 2009.

[14] M.Perez, L.Brunaud, S.Kedaifa, C.Guillotin, A.Gerardin, D.Quilliot. Does anatomy explain the origin of a leak after sleeve gastrectomy? Obes Surg; 24, Vol. PP. (1717-17237), 2014.

[15] F.Benedix, D.Benedix, C.Knoll, R. Weiner, C.Bruns, T.Manger, C.Stroh. Obesity Surgery Working Group. Are there risk factors that increase the rate of staple line leakage in patients undergoing primary sleeve gastrectomy for morbid obesity? Obes Surg; Vol. 24, PP. (1610-1616), 2014.

[16] T.Palao Garcia, T. J. Small arteries, big problems: New insights in the mechanisms of small artery remodeling in hypertension, 2018.

[17] C.Boeker, J.Mall, C.Reetz, K.Yamac, L.Wilkens, C.Stroh, H.Koehler. Laparoscopic sleeve gastrectomy: investigation of fundus wall thickness and staple height - an observational cohort study. Obes Surg; Vol. 27, PP. (3209-3214), 2017.

[18] N.Sakran, D.Goitein, A.Raziel, A.Keidar N.Beglaibter N. Gastric leaks after sleeve gastrectomy: a multicenter experience with 2,834 patients. Surg Endosc; Vol. 27, PP. (240-245), 2013.

[19] P.Noe, M.Nedelcu, M.Gagner. Impact of the surgical experience on leak rate after laparoscopic sleeve gastrectomy. Obes Surg; vol. 26, PP. (17821787), 2016.

[20] M.Sajid, K.Khatri, K.Singh. Use of staple-line reinforcement in laparoscopic gastric bypass surgery: a meta-analysis. Surg Endosc.; Vol. 25, PP. (288491), 2011.

[21] H.Wang, J.Lu, J.Feng, Z.Wang Z. Staple line oversewing during laparoscopic sleeve gastrectomy. The Annals of The Royal College of Surgeons of England; Vol. 99(7), PP. (509-514), 2017.

[22] M.Sepúlveda, C.Astorga, J.Hermosilla, M. Alamo. Staple line reinforcement in laparoscopic sleevegastrectomy: experience in 1023 consecutive cases. Obesity surgery; Vol. 27(6), PP. (1474-80), 2017. 